\title{
OPEN Split NanoLuc technology allows quantitation of interactions between PII protein and its receptors with unprecedented sensitivity and reveals transient interactions
}

\author{
Rokhsareh Rozbeh \& Karl Forchhammer ${ }^{\bowtie}$
}

PII proteins constitute a widespread signal transduction superfamily in the prokaryotic world. The canonical PII signal proteins sense metabolic state of the cells by binding the metabolite molecules ATP, ADP and 2-oxoglutarate. Depending on bound effector molecule, PII proteins interact with and modulate the activity of multiple target proteins. To investigate the complexity of interactions of PII with target proteins, analytical methods that do not disrupt the native cellular context are required. To this purpose, split luciferase proteins have been used to develop a novel complementation reporter called NanoLuc Binary Technology (NanoBiT). The luciferase NanoLuc is divided in two subunits: a 18 kDa polypeptide termed "Large BiT" and a 1.3 kDa peptide termed "Small BiT", which only weakly associate. When fused to proteins of interest, they reconstitute an active luciferase when the proteins of interest interact. Therefore, we set out to develop a new NanoBiT sensor based on the interaction of PII protein from Synechocystis sp. PCC6803 with PII-interacting protein X (PipX) and N-acetyl-Lglutamate kinase (NAGK). The novel NanoBiT sensor showed unprecedented sensitivity, which made it possible to detect even weak and transient interactions between PII variants and their interacting partners, thereby shedding new light in PII signalling processes.

PII signalling proteins are ubiquitous in nature, in particular in Prokaryotes and plastids of Archaeplastida ${ }^{1}$. Their general task is to sense the metabolic state of the cells by binding in an interactive manner the metabolite status reporter molecules ATP and ADP as well as 2-oxoglutarate ${ }^{2}$. Effector molecule binding results in different conformational states of PII proteins, in particular, of their large flexible T-loops acting as versatile protein-protein interaction modules. Depending on the metabolite binding status, and thus, the PII-conformation, various PII receptors read out the metabolic information processed by PII. This is achieved through transient interactions with PII, which alter the activities of the PII-receptor proteins. Although initially thought to be confined to the regulation of nitrogen metabolism, research in the past years has unveiled a plethora of PII regulated proteins ${ }^{2,3}$.

The focus of our work has been on the PII regulated processes in cyanobacteria. These are oxygenic photosynthetic, autotrophic bacteria playing important roles in global carbon- and nitrogen cycles. The first PII interaction partners, the N-acetyl-L-glutamate kinase (NAGK), a key enzyme of arginine synthesis, and PipX, a transcriptional co-activator of the global nitrogen control transcription factor NtcA, were identified by yeasttwo hybrid screening ${ }^{4,5}$. Detailed mechanistic insight in the interaction of PII with these receptors has been obtained by solving the X-ray crystallographic structures of the proteins in complex ${ }^{6,7}$, and characterizing the PII interactions mainly through Surface-Plasmon-Resonance analysis ${ }^{8-11}$. Even more details have been obtained by FRET-based quantitative analysis by fusing PII and NAGK or PipX to fluorescent proteins ${ }^{12-14}$.

More recently, mainly through PII-co-purification approaches, novel interaction partners of PII proteins have been identified in cyanobacteria, such as the biotin carboxyl carrier protein (BCCP)-subunit of the AcetylCoA carboxylase ${ }^{15}$, an ensemble of nitrogen uptake systems (the nitrate transport system NRT, urea-transport 
system URT and ammonium transport protein AMT1 ${ }^{16}$, the Phosphoenolpyruvate carboxylase (PepC) ${ }^{17}$ and two regulatory peptides, termed PirC and PirA, the former regulating carbon flow through interaction with the Phosphoglycerate mutase (PGAM), and the second, the arginine synthesis pathway ${ }^{18,19}$. However, for all the latter interaction partners, structural information is not yet available. Of the recently identified PII-receptors, in vitro protein interaction analysis has been performed for BCCP, PEPC, PirC and PirA. A striking feature of PII-PII-receptor interactions is in the ability of the same effector molecule to differentially affect the interaction of PII with different receptors. For example, PII binds NAGK in the absence of effector molecules. Binding of ADP to PII increases complex dissociation ${ }^{10}$, and ATP in combination with 2-OG completely prevents complex formation ${ }^{20}$. By contrast, ADP enhances the stability of the PII-PipX complex, whereas ATP together with 2-OG again prevents complex formation. When small amounts of ADP were added to Mg-ATP/2-OG pre-loaded PII, PipX was again able to bind to PII, whereas small amounts of ADP did not affect PII-NAGK interactions in presence of $\mathrm{ATP}^{10}$. Structural analysis provided a mechanistic explanation: Formation of the PII-PipX complex stabilizes the conformation of PII that preferentially binds ADP. By contrast, PII in the NAGK complex adopts a conformation that preferentially binds ATP but is incompatible with 2-OG binding ${ }^{11}$. As a consequence, interaction between PII and PipX is highly sensitive to fluctuations in the ATP/ADP ratio whereas PII -NAGK complex responds mainly to changes to the 2-oxoglutarate levels, as long as sufficient ATP is present. This allows PipX to sense the energy status via PII interaction whereas at the same time, NAGK can read out the level of 2-oxoglutarate (a status reporter of the carbon/nitrogen balance), provided that PII is present in excess over the receptors, which appears to be the case $\mathrm{e}^{21}$.

A limitation of the interaction analyses using Surface-Plasmon-Resonance (SPR) or Biolayer interferometry (BLI) comes from the fact that one interaction partner has to be immobilized on a sensor-surface, restricting access and mobility of the proteins. Indeed, fixing PII via a C-terminal Strep-tag to the surface of a Biacore sensor disturbed the response of the PII-PipX complex to ATP and 2-oxoglutarate ${ }^{10}$. Likewise, the use of FRET based analytics requires the fusion of large fluorescence proteins to both interaction partners and is highly sensitive to background fluorescence ${ }^{22}$. An alternative is the use of protein fragment complementation assays (PCA). PCA is a powerful approach to determine protein-protein interactions and has been applied in various variants ${ }^{23}$. A very attractive PCA version that was recently developed to determine PII protein interactions in solution is the split NanoLuc system. NanoLuc is an engineered luciferase enzyme from the deep ocean shrimp with exceptionally bright bioluminescence ${ }^{24}$. A variant of the enzyme was further engineered as protein fragment complementation reporter ${ }^{25}$, now commercially available as NanoLuc Binary Technology (NanoBiT) ${ }^{26}$. The small 11 amino acids fragment (termed Small BiT, SmBiT) was constructed in a way that it has low intrinsic affinity $(190 \mu \mathrm{M})$ towards the large complementation fragment (Large BiT, LgBiT). When LgBiT and SmBiT fragments are fused to candidate interacting proteins, luminescence is only restored when the candidate proteins interact with each other.

Recently, the NanoBiT technology was used to quantitatively analyse several protein-protein interactions in mammalian cells. It was used to verify the interaction between SME1 $\beta$-lactamase and a set of inhibitor binding proteins in vivo with the optimal performance when LgBiT was attached to the C-terminus of SME1 and $\mathrm{SmBiT}$ was attached to the C-terminus of inhibitor binding proteins ${ }^{25}$. The system was also used to accurately characterize the interaction between bacterial transcription factors NusB and NusE, was well as the interaction between RNA polymerase and $\sigma$ A from Bacillus subtilis ${ }^{27}$. Here, we employed NanoBiT technology to analyse the interactions of PII with PipX and NAGK, and show that this method provides superior quantitative results with exceptionally high sensitivity in comparison to SRP or BLI. These analyses also provide additional insights into the sophisticated network of PII interactions.

\section{Results}

Development of the NanoBiT sensor. Initially we constructed hybrid PII fusion proteins that contained LgBiT fragment at the C-terminus and SmBiT fragments were fused to the tip of the T-loop with three different linkers (24aa linker, 16aa linker and 8aa linker) in a similar manner than previously reported for hybrid PII-FRET constructs ${ }^{22}$. Of these constructs, only the 16 aa linker construct showed a response to the addition of PII effector molecules, however with only a response of $30 \%$ signal difference (data not shown). Therefore, we decided to separate the NanoBiT fragments in two distinct polypeptites: on PII and a PII-receptor protein, using the 16 aa linker to fuse the SmBiT fragment, which appeared most suitable from the initial experiments. The LgBiT fragment was fused at the C-terminally located Strep-tag purification linker of PII, which was already successfully used as linker for PII-VENUS FRET constructs ${ }^{12}$. The SmBiT fragment was fused at the C-terminus of PipX, with a 16 amino acids flexible linker in between (Fig. 1a). The gene for the PipX-SmBiT construct was cloned into expression vector pTEV5, containing an N-terminal $\mathrm{His}_{6}$ affinity tag. When expressed in E.coli the recombinant proteins formed inclusion bodies. These were dissolved in $8 \mathrm{M}$ urea for His-tag affinity purification, and the purified proteins were subsequently re-folded. Purified, refolded PipX-SmBiT and PII-LgBiT alone showed low levels of background luminescence $\sim 1 \times 10^{-5} \mathrm{RLU}$ (relative light unit). This background was subtracted from the luminescence signals of the subsequent assays. In initial trials, PipX-SmBiT was used at a concentration of $10 \mathrm{nM}$ and PII-LgBiT was titrated at increasing concentrations starting with $0.33 \mathrm{pM}$ in a buffer containing $2 \mathrm{mM}$ ADP, which are the conditions that were previously shown to be optimal for PII-PipX interaction ${ }^{10}$. After $15 \mathrm{~min}$ of incubation at $30^{\circ} \mathrm{C}$, the luminescence signal was recorded and plotted against the respective PII-LgBiT concentration. As shown in Fig. 1b, at low PII-LgBiT concentrations, a linear increase of luminesce up to $1 \times 10^{7} \mathrm{RLU}$ was detected (corresponding to $10 \mathrm{pM}$ PII) whereas the curved flattened at higher concentration of PII-LgBiT. This indicates that the higher concentrations of NanoLuc are beyond the linear detection range of the assay. Therefore, in all subsequent assays the concentration of PII-LgBiT was limited to $10 \mathrm{pM}$. 
$\mathbf{a}$
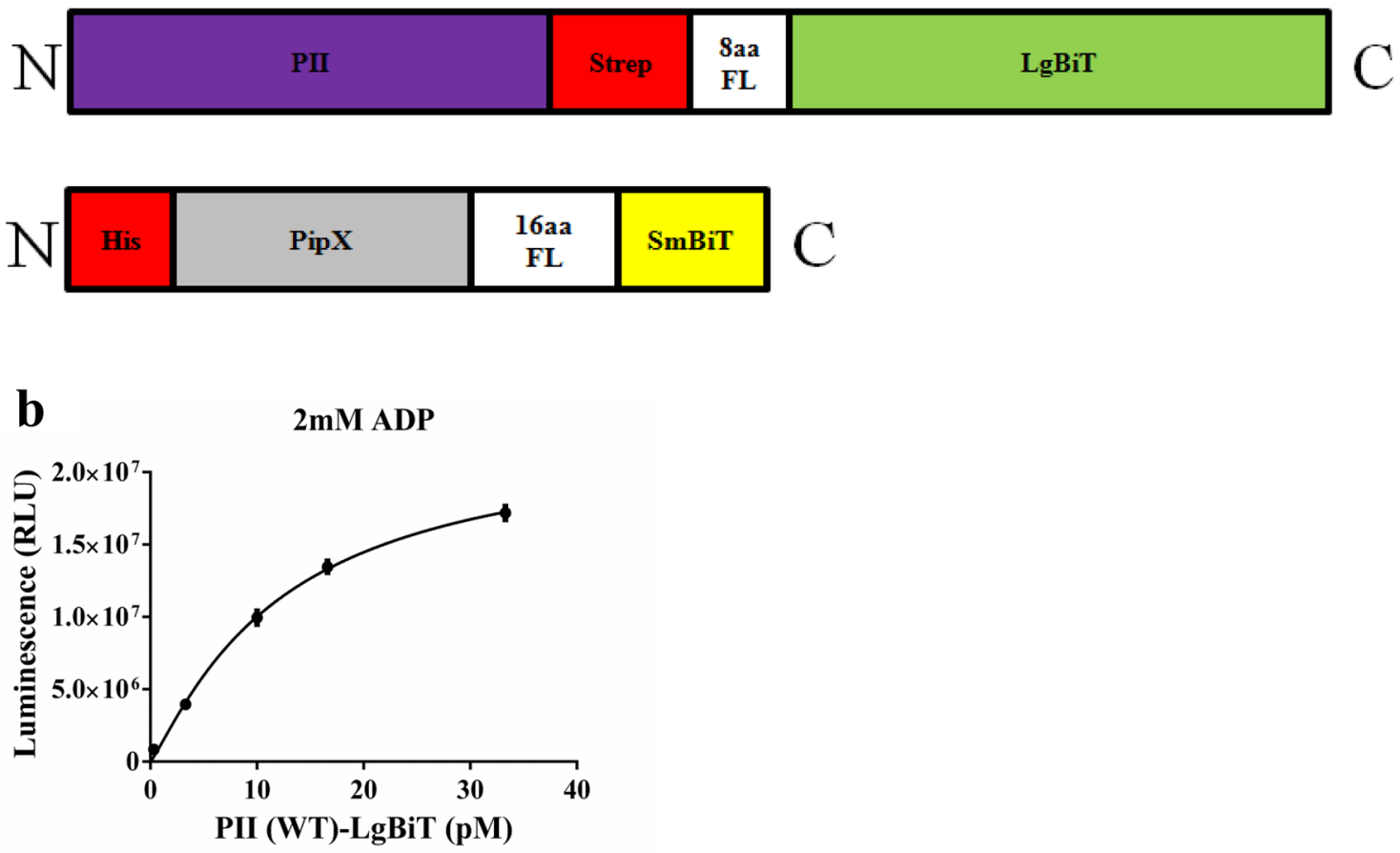

Figure 1. Fusion protein constructs and NanoBiT responce. (a) Schematic representation of PII-LgBiT and PipX-SmBiT fusion proteins. Flexible linker with 16 amino acids (16aa) in construct fused to SmBiT. (b) Titration of PII-LgBiT (0.33-33 pM) with constant amount of PipX-SmBiT (10 nM) in the presence of $2 \mathrm{mM}$ ADP. Graph represents mean \pm SD of three independent experiments.

PII-PipX binding kinetics and influence of effector molecules. The next step was to monitor the kinetics of PII-PipX complex formation in real-time by continuously measuring the luminescence in the presence of various PII effector molecules. Therefore, PII-LgBiT (concentration of $10 \mathrm{pM}$ trimer) was first mixed with the indicated amounts of PipX-SmBiT and luminescence was measured for five minutes without effector molecules. As shown in Fig. 2a,b, this resulted only in a very low increase in luminescence. Subsequent addition of adenyl-nucleotides immediately stimulated the interaction as shown by the rise of luminescence signal (Fig. 2a,b). With $2 \mathrm{mM}$ ATP, complex formation reached a plateau after about $10 \mathrm{~min}$, indicating equilibrium between complex association and dissociation. In presence of ADP, the increase was much steeper, and $10 \mathrm{~min}$ after ADP addition, equilibrium was not yet reached, with a maximal signal for the ADP-promoted complex 2,5 times higher than that of the ATP-promoted complex.

To quantitatively determine the dissociation constant of PII-PipX interaction in presence of $2 \mathrm{mM}$ ATP or ADP, increasing concentrations of PipX were titrated to a fixed concentration of PII-LgBiT (10 pM trimer). Then, the RLUs were plotted against the PipX-SmBiT concentrations (Fig. 2c). From these curves, $\mathrm{K}_{\mathrm{D}}$ values were calculated assuming that in equilibrium the dissociation constant follows the Eq. (1):

$$
\mathrm{K}_{\mathrm{D}}=[\mathrm{PII}-\mathrm{LgBiT}] \times[\mathrm{PipX}-\mathrm{SmBiT}] /[\mathrm{PII}-\operatorname{LgBiT} * \operatorname{PipX}-\mathrm{SmBiT}] .
$$

At the concentration of PipX-SmBiT at which half maximal luminescence is observed, $50 \%$ of PII should be in the PipX complex. This concentration should therefore correspond to the $\mathrm{K}_{\mathrm{D}}$ of the complex (provided that PipX is in large excess over PII so that the free PipX concentrations approximately equal the total PipX concentration). We estimated the half-maximal RLU value by hyperbolic fitting, resulting in a $K_{D}$ of $5.7 \pm 0.78 \mathrm{nM}$ for the PII-PipX complex in presence of ADP and of $52.4 \pm 0.92 \mathrm{nM}$ in presence of ATP (Table 1).

The ATP to ADP ratio is an indicator of the cellular energy state. To determine its impact on PII-PipX interaction as revealed by NanoBiT analysis, the real-time luminescence increase was recorded at various ATP/ADP ratios, keeping the total $\mathrm{ADP}+\mathrm{ATP}$ concentration constant at $2 \mathrm{mM}$. As shown in Fig. $2 \mathrm{~d}$, ADP is the dominant effector over ATP. Already $0.5 \mathrm{mM}$ ADP in presence of $1.5 \mathrm{mM}$ ATP (ATP/ADP ratio 3 ) is sufficient to increase luminescence signal up to $80 \%$. The result reported in this study matches a previous similar experiment performed by SPR, which also showed that the interaction between PII and PipX is highly sensitive to fluctuations in the ATP/ADP ratios ${ }^{11}$. Strongly fluctuating ATP to ADP levels in Synechocystis cells have been reported in particular in response to light - dark shifts or different nutritional conditons ${ }^{28-30}$, Therefore, the response of PII-PipX interaction to differing ATP-ADP ratios seems physiologically relevant and matches the observations reported by Espinosa et $\mathrm{al}^{29}$.

Next, we studied the effect of different 2-OG concentrations on PII-PipX complex formation (10 pM PII and $10 \mathrm{nM}$ PipX) in the presence of $2 \mathrm{mM}$ ATP. First, the mixtures of PII-LgBiT and PipX-SmBiT were incubated with the various 2-OG concentrations in the absence of ATP. After $5 \mathrm{~min}, 2 \mathrm{mM}$ ATP was added and complexes 

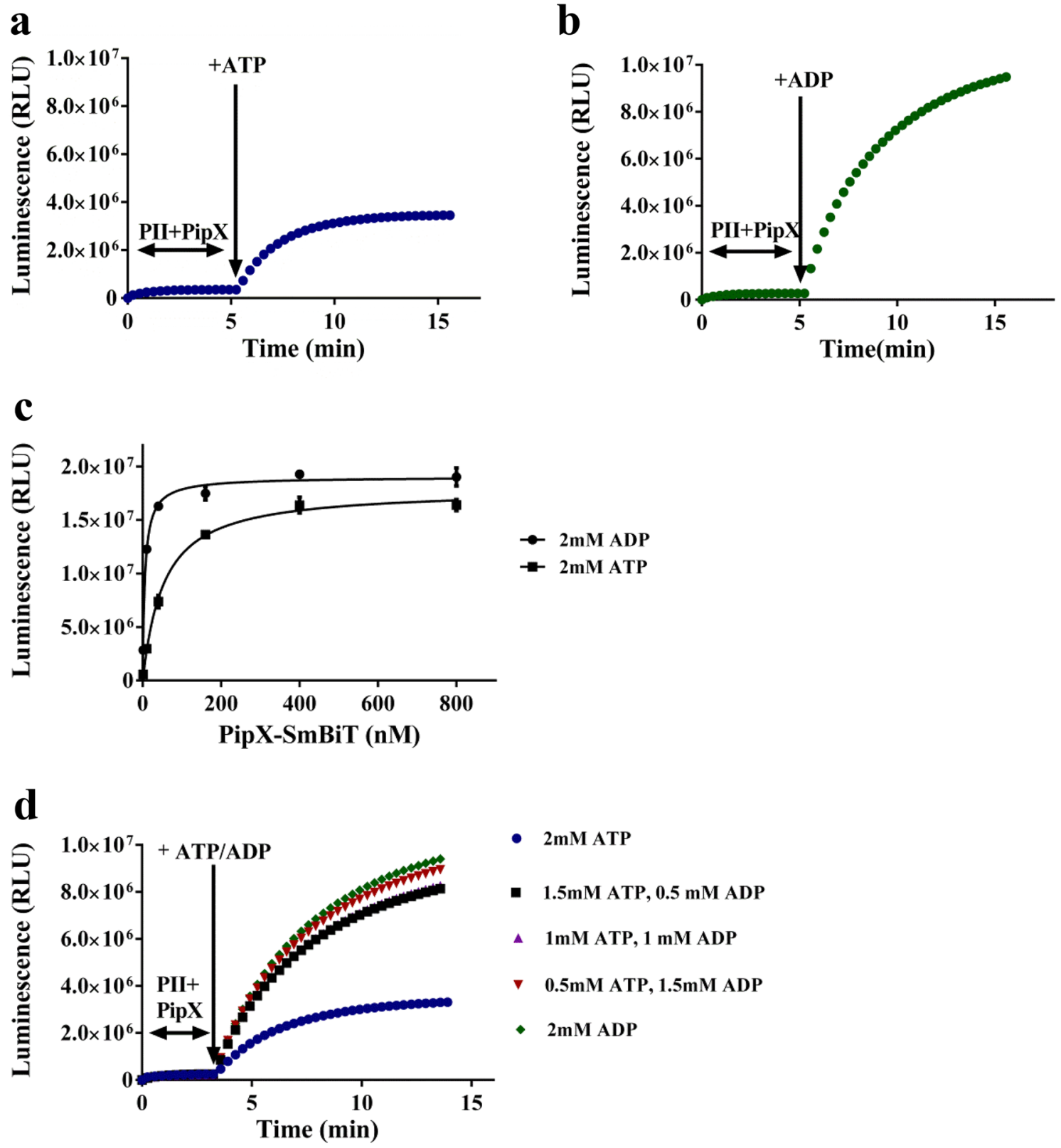

Figure 2. Effect of ATP or ADP on PII-LgBiT-PipX-SmBiT complex formation. (a and $\mathbf{b})$ Time course of complex formation after adding 2 mM ATP (a) or 2 mM ADP (b). (c) Titration of PipX-SmBiT (0, 1, 10, 40, 160, $400,800 \mathrm{nM})$ to PII-LgBiT (10 pM) in the presence of $2 \mathrm{mM}$ ATP or $2 \mathrm{mM}$ ADP. (d) Time course of complex formation PII-LgBiT (10 pM) and PipX-SmBiT (10 nM) in the presence of different ATP/ADP mixtures at a total ADP + ATP concentration of $2 \mathrm{mM}$. Graphs represent mean $\pm \mathrm{SD}$ of three independent experiments.

\begin{tabular}{|c|c|c|c|c|c|c|c|c|c|c|}
\hline \multirow[b]{2}{*}{$\begin{array}{l}\text { Protein } \\
\text { complex }\end{array}$} & \multicolumn{10}{|c|}{ Effector molecules condition and $K_{D}(n M)$ of protein complex } \\
\hline & $2 \mathrm{mM}$ АTP & $2 \mathrm{mM}$ ADP & No effector & $\begin{array}{l}2 \mathrm{mM} \text { ATP/ } \\
2 \mathrm{mM} \text { Arg }\end{array}$ & $\begin{array}{l}\text { 2mMATP/ } \\
\text { 2mMNAG }\end{array}$ & $\begin{array}{l}2 \mathrm{mM} \text { ATP/ } \\
50 \mathrm{mM} \text { NAG }\end{array}$ & $\begin{array}{l}2 \mathrm{mM} \text { ATP/ } \\
2 \mathrm{mM} \text { Arg/ } \\
2 \mathrm{mM} \text { NAG }\end{array}$ & $\begin{array}{l}2 \mathrm{mM} \text { ATP/2 mM } \\
\text { Arg/50 mM NAG }\end{array}$ & $\begin{array}{l}2 \mathrm{mM} \text { ADP/ } \\
2 \mathrm{mM} \text { NAG }\end{array}$ & $\begin{array}{l}2 \mathrm{mM} \text { ADP/ } \\
50 \mathrm{mM} \text { NAG }\end{array}$ \\
\hline $\begin{array}{l}\text { PII (WT)- } \\
\text { NAGK }\end{array}$ & $8.8 \pm 0.72$ & $30.8 \pm 0.12$ & $12.9 \pm 0.65$ & $17.8 \pm 0.72$ & $4.7 \pm 0.35$ & $0.74 \pm 0.081$ & $16.5 \pm 1.08$ & $9.5 \pm 0.20$ & $14.9 \pm 1.13$ & $1.2 \pm 0.053$ \\
\hline $\begin{array}{l}\text { PII(S49E)- } \\
\text { NAGK }\end{array}$ & $155 \pm 0.37$ & $95.3 \pm 1.05$ & $133 \pm 0.62$ & $432 \pm 1.34$ & $10.7 \pm 0.91$ & $3.39 \pm 0.73$ & $\mathrm{~nm}$ & $\mathrm{~nm}$ & $\mathrm{~nm}$ & $\mathrm{~nm}$ \\
\hline $\begin{array}{l}\text { PII (I86N)- } \\
\text { NAGK }\end{array}$ & $7 \pm 0.20$ & $11.7 \pm 0.14$ & $12.9 \pm 0.20$ & $1.56 \pm 0.48$ & $4.1 \pm 0.33$ & $0.68 \pm 0.031$ & $\mathrm{~nm}$ & $\mathrm{~nm}$ & $\mathrm{~nm}$ & $\mathrm{~nm}$ \\
\hline $\begin{array}{l}\text { PII (WT)- } \\
\text { PipX }\end{array}$ & $52 \pm 0.92$ & $5.7 \pm 0.78$ & nd & $\mathrm{nm}$ & $\mathrm{nm}$ & $\mathrm{nm}$ & $\mathrm{nm}$ & $\mathrm{nm}$ & $\mathrm{nm}$ & $\mathrm{nm}$ \\
\hline
\end{tabular}

Table 1. Dissociation constants (nM) of PII-LgBiT fusion variants with NAGK-SmBiT and PipX-SmBiT in presence of different metabolites. Data are the mean \pm SD of triplicate measurements. The raw data was fitted using one site-specific binding with hill slope. $n d$ not detectable; $n m$ : not measured 

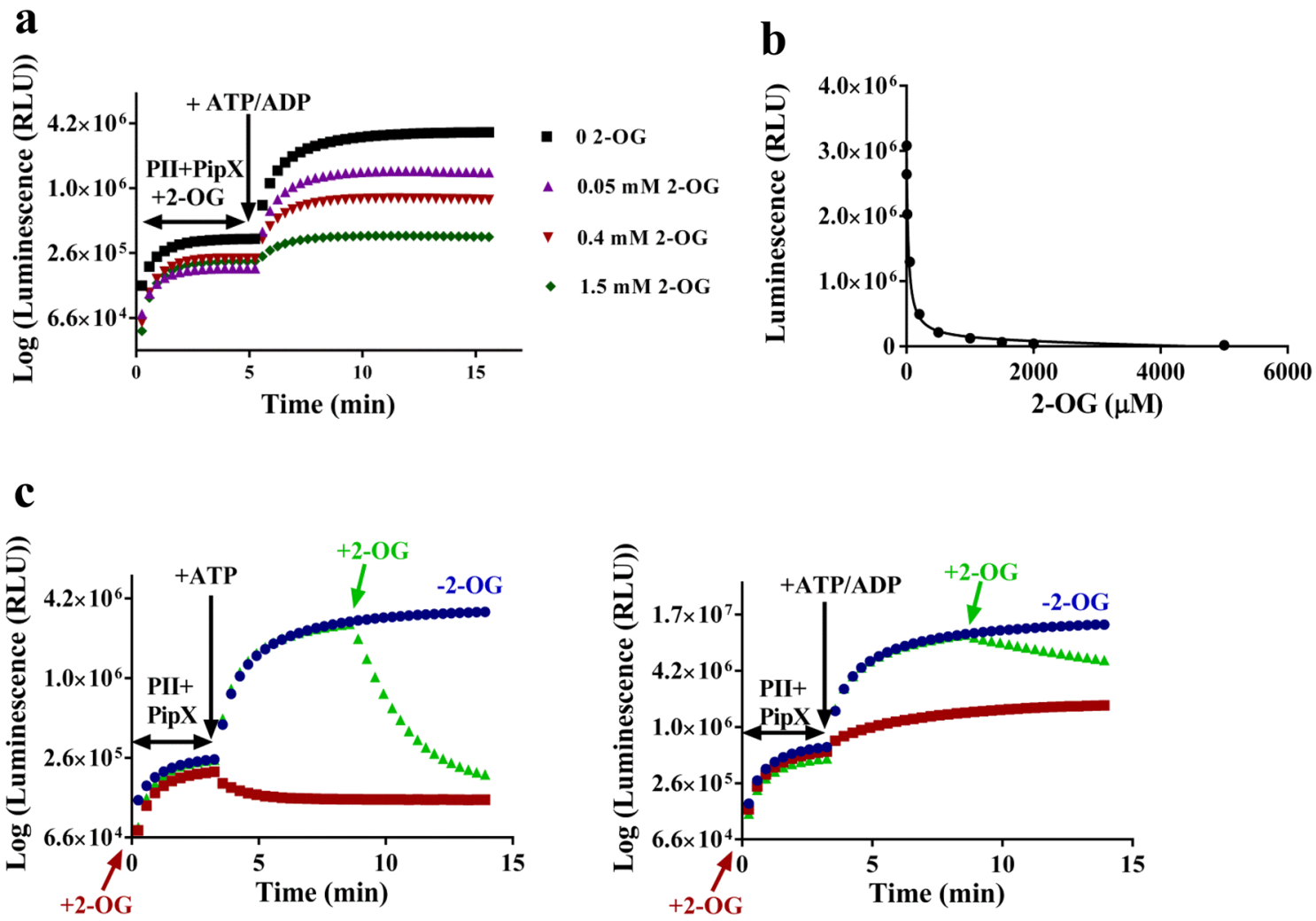

Figure 3. Effect of 2-OG on PII-LgBiT (10 pM) and PipX-SmBiT (10 nM) complex formation. (a) Interaction of PII-LgBiT and PipX-SmBiT in the presence of: none, 0, $0.05 \mathrm{mM}, 0.4 \mathrm{mM}$ and $1.5 \mathrm{mM}$ 2-OG. (b) Quantitative analysis of 2-OG dependent inhibition of complex formation. (c) Time course of complex dissociation after addition of $2 \mathrm{mM} 2-\mathrm{OG}$ at indicated time point (green symbols). Control without 2-OG addition (blue symbols). First, PII-LgBiT and PipX-SmBiT were incubated without effectors (blue and green) or with only 2-OG (red symbols). After $4 \mathrm{~min}$, either ATP (left) or $1 \mathrm{mM}$ ATP and $1 \mathrm{mM} \mathrm{ADP}$ (right) were added, followed by the addition of $2-\mathrm{OG}$.

formed until reaching saturation after $15 \mathrm{~min}$ (Fig. 3a). To quantify the effect, the maximal RLU values were plotted against the respective $2-\mathrm{OG}$ concentrations $(0,0.002,0.01,0.05,0.2,0.5,1,1.5,2,5 \mathrm{mM})$ in the assay (Fig. 3b). In a similar way as reported by SPR, increasing 2-OG concentrations gradually prevented complex formation. Curve fitting resulted in an $\mathrm{IC}_{50}$ of $25 \mu \mathrm{M}$ (corresponding to a $50 \%$ signal drop) for the inhibition by 2 -OG (Fig. 3b). This value is in a similar range, albeit $40 \%$ lower than the average $\mathrm{K}_{\mathrm{D}}$ of 2 -OG binding to PII$\operatorname{Mg}$-ATP $(39 \mu \mathrm{M})^{10}$, whereas the $\mathrm{K}_{\mathrm{D}}$ of 2 -OG binding to the first (highest affinity) binding site in trimeric PII is approx. $5 \mu \mathrm{M}$. This result indicates that trimeric PII with the highest affinity site occupied by 2-OG can still bind PipX via its free subunits, and only when fully occupied by 2-OG, PII is impaired in PipX binding.

To study the kinetics of 2-OG promoted PII-PipX complex dissociation with the NanoBiT technology, the luminescence signal dynamics was recorded in time-course experiments in which effector molecules were added successively (Fig. 3c). First, PII-LgBiT and PipX-SmBiT were pre-incubated without adenyl-nucleotides (green and blue symbols) or in a control experiment, in the presence of $2 \mathrm{mM} \mathrm{2-OG} \mathrm{(red} \mathrm{symbols).} \mathrm{After} \mathrm{four} \mathrm{min-}$ utes, $2 \mathrm{mM}$ ATP (left panel) or $1 \mathrm{mM}$ ATP $+1 \mathrm{mM}$ ADP (right panel) were added to allow complex formation. Five minutes later, $2 \mathrm{mM}$ 2-OG was added to one sample each (green symbols) and the luminescence signal was recorded for further five minutes. In the presence of ATP alone, 2-OG completely dissociated the complex within two minutes to basal levels. After $45 \mathrm{~s}$, about half of the complex was dissociated. A different response was observed in the presence of $1 \mathrm{mM}$ ATP $+1 \mathrm{mM}$ ADP, where dissociation was strongly mitigated. It required approximately $340 \mathrm{~s}$ to dissociate half of the complexes to basal level, 7-8-times longer than in the absence of ADP. The $\mathrm{K}_{\mathrm{D}}$ of 2-OG binding to PII in presence of $1 \mathrm{mM}$ ATP $+1 \mathrm{mM}$ ADP was previously determined to be $180 \mu \mathrm{M}$, compared to about $39 \mu \mathrm{M}$ in presence of $2 \mathrm{mM}$ ATP ${ }^{10}$. Therefore, the slower dissociation of the PII-PipX complex is in accord with the decreased affinity of PII for 2-OG in presence of $1 \mathrm{mM}$ ATP/ADP.

PII-NAGK binding kinetics and influence of effector molecules. To find out whether NanoBiT can also be applied to directly study PII interaction with other receptor N-acetyl-L-glutamate kinase (NAGK), the second well-characterized cyanobacterial PII-receptor, a PII-LgBiT-NAGK-SmBiT pair was developed. Therefore, the SmBiT fragment together with the 16 amino acids flexible linker was fused to the C-terminus of NAGK (Fig. 4a). As a first experiment, the purified NAGK-SmBiT fusion protein was titrated to $10 \mathrm{pM}$ PII-LgBiT in the presence of $2 \mathrm{mM}$ ATP, $2 \mathrm{mM}$ ADP or in the absence of effector molecules (Fig. $4 \mathrm{~b}$ ). Efficient complex formation 
$\mathbf{a}$


Figure 4. NanoBiT pair consisting of PII-LgBiT-NAGK-SmBiT. (a). Schematic representation of NanoBiTsensor construct as indicated (b) Titration of NAGK-SmBiT in the range of 3-333 nM with 10 pM PII-LgBiT in the presence of $2 \mathrm{mM}$ ATP or $2 \mathrm{mM}$ ADP or in the absence of effector molecules. (c) Inhibitory effect of 2-OG $(0,0.01,0.05,0.2,0.5,1,2,5 \mathrm{mM})$ on PII-LgBiT $(10 \mathrm{pM})$ and NAGK-SmBiT $(27 \mathrm{nM})$ complex formation in the presence of $2 \mathrm{mM}$ ATP. Graphs represent mean \pm SD of three independent experiments.

was detected between PII-LgBiT and NAGK-SmBiT in the absence of effector molecules and in presence of ATP. Complex formation also occurred in the presence of ADP, albeit with lower affinity (Fig. 4b). Hyperbolic fitting resulted in apparent $\mathrm{K}_{\mathrm{D}}$ values of $12.9 \pm 0.65 \mathrm{nM}$ in the absence of effectors, $8.8 \pm 0.72 \mathrm{nM}$ in the presence of ATP and $30.8 \pm 0.12 \mathrm{nM}$ in the presence of ADP. In SPR experiments, only weak and transient PII-NAGK complexes could be detected in presence of ADP, which prevented quantitative determination ${ }^{10}$. This result demonstrates superior sensitivity of the NanoBiT over SPR for the detection and quantification of weak interactions.

To monitor the effect of 2-OG on PII-NAGK complex formation, 10 pM PII-LgBiT trimer was incubated with $160 \mathrm{nM}$ NAGK-SmBiT (26.6 nM hexameric NAGK) in the presence of $2 \mathrm{mM}$ ATP and different concentrations of 2-OG. Preliminary experiments showed that to achieve equilibrium, at least 30 min incubation time was necessary. Therefore, for quantification of 2-OG effects, the luminescence reagent was added $40 \mathrm{~min}$ after the start of PII-LgBiT-NAGK-SmBiT complex formation. Then, luminescence was recorded and plotted against the 2-OG concentrations (Fig. 4c). Similar to what observed for the PII-PipX complex, the inhibitory effect of 2 -OG was clearly revealed by the NanoBiT assay. The $\mathrm{IC}_{50}$ for $2-\mathrm{OG}$, which led to $50 \%$ drop in luminescence, was determined to be $0.15 \mathrm{mM}$. This value correlated well with previous data obtained through different experimental approaches. Determination of PII-NAGK interaction by FRET assay revealed an $\mathrm{IC}_{50}$ for 2 -OG of $0.1 \mathrm{mM}^{12}$, and SPR and enzyme assays yielded an $\mathrm{IC}_{50}$ of $0.12 \mathrm{mM}^{20}$.

Competition between PipX and NAGK for binding to PII. Another possible application of the Nano$\mathrm{BiT}$ system was to reveal competition between PipX and NAGK for PII binding. In a first experiment, PipX was titrated to a constant concentration of PII-LgBiT and NAGK-SmBiT (10 pM trimer and $180 \mathrm{nM}$ monomer, respectively) in the presence of $2 \mathrm{mM}$ ADP. As expected from higher affinity of PII to PipX than to NAGK in presence of ADP (compare Tab.1), the addition of PipX completely abolished luminescence of the PII-LgBiTNAGK-SmBiT complex (Fig. 5a left panel). Vice versa, addition of increasing NAGK amounts to a constant concentration of PII-LgBiT and PipX-SmBiT in presence of ADP showed no effect on the luminescence signal, clearly indicating that in presence of ADP, PII exclusively binds to PipX, when both partners are present (Fig. 5a, right panel). A more complicated situation was observed when the same set of experiments was performed in presence of ATP. Titrating PipX to the PII-LgBiT and NAGK-SmBiT sensor pair resulted in a gradual decrease in luminescence. Although the affinity of PII to PipX is lower than towards NAGK in presence of ATP, the addition of a 1:1 stoichiometric amount of PipX to NAGK reduced the luminescence signal to about $50 \%$ and higher amounts of PipX decreased the signal further (Fig. 5b, left panel). Conversely, the PII-LgBiT and PipX-SmBiT sensor pair was quite resistant towards the addition of NAGK. Even a large excess of NAGK over PipX reduced the signal from the PII-LgBiT and PipX-SmBiT sensor pair only partially, although at these high NAGK concentrations, PII is expected to preferentially interact with NAGK (Fig. 5b, right panel). A possible explanation could be a weak interaction of PipX-SmBiT protein to PII-LgBiT-NAGK complexes, resulting in luminescence signals, due to the high sensitivity of the reporter system. 
$\mathbf{a}$

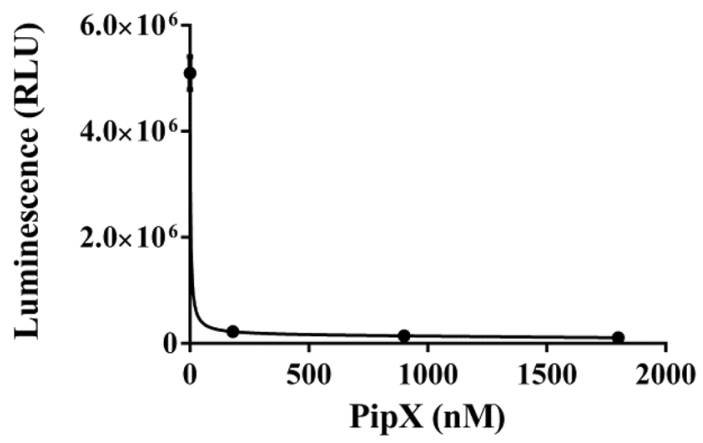

b

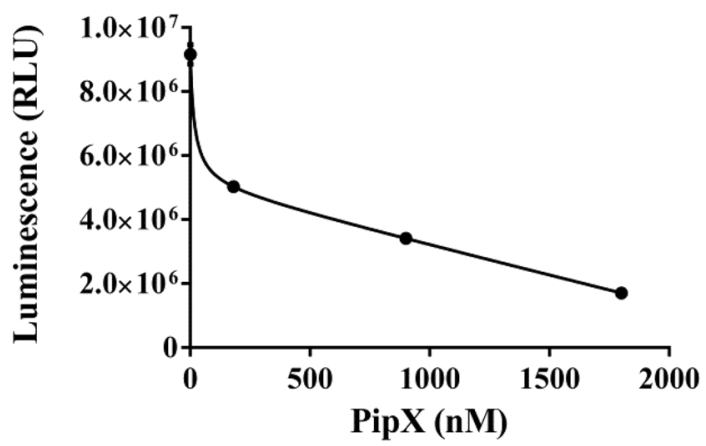

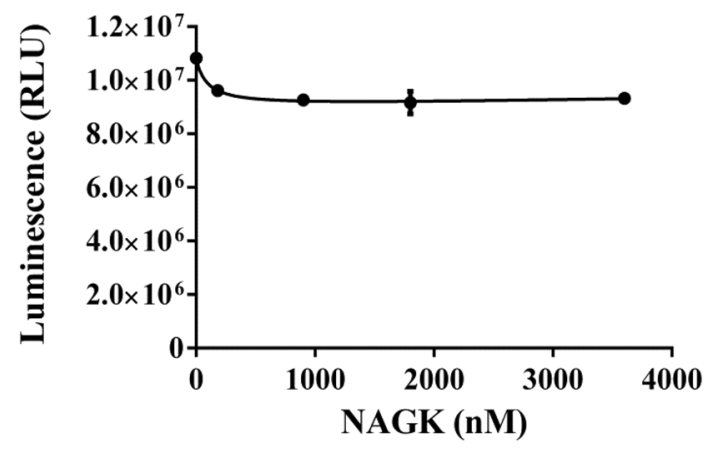

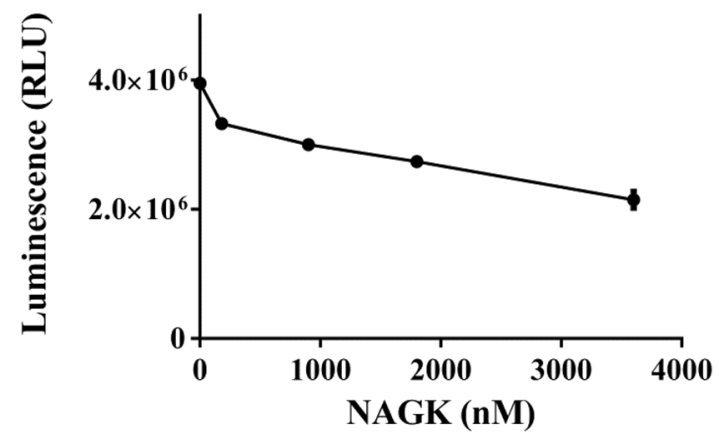

Figure 5. Competition between PII interaction partners to bind to PII in the presence of $2 \mathrm{mM}$ effector molecules: (a, left panel) Competition between NAGK-SmBiT and PipX for PII-LgBiT binding in the presence of $2 \mathrm{mM}$ ADP: The interaction between $10 \mathrm{pM}$ PII-LgBiT (trimer) and $180 \mathrm{nM}$ NAGK-SmBiT (monomer) was competed with increasing concentrations of PipX (180, 900 and $1800 \mathrm{nM})$ monomer. (a, right panel) Competition between NAGK and PipX-SmBiT for PII-LgBiT binding in the presence of $2 \mathrm{mM}$ ADP: The interaction between $10 \mathrm{pM}$ PII-LgBiT trimer and $10 \mathrm{nM}$ PipX-SmBiT monomer was competed with the addition of increasing concentrations of $\operatorname{NAGK}(180,900,1800$ and $3600 \mathrm{nM})$ monomer. (b, left panel) Competition between NAGK-SmBiT and PipX for PII-LgBiT binding in the presence of $2 \mathrm{mM}$ ATP: The interaction between 10 pM PII-LgBiT (trimer) and $180 \mathrm{nM}$ NAGK-SmBiT (monomer) was competed with increasing concentrations of PipX (180, 900 and $1800 \mathrm{nM})$ monomer. (b, right panel) Competition between NAGK and PipX-SmBiT for PII-LgBiT binding in the presence of $2 \mathrm{mM}$ ATP: The interaction between $10 \mathrm{pM}$ PII-LgBiT trimer and $50 \mathrm{nM}$ PipX-SmBiT monomer was competed with the addition of increasing concentrations of NAGK $(180,900,1800$ and $3600 \mathrm{nM})$ monomer. Graphs represent mean \pm SD of two independent experiments.

Affinity of NAGK to PII variants in the presence of effector molecules. The association kinetics of NAGK-SmBiT was analysed with two mutant PII-LgBiT variants: The T-loop variant PII-S49E was shown previously to be strongly impaired in NAGK interaction ${ }^{6,12}$, whereas by contrast, the PII-I86N variant was identified as a hyperactive NAGK binder ${ }^{20}$. As shown in Fig. 6a-c, the NanoBiT assay was able to reveal low residual binding of the phosphomimetic variant PII-S49E to NAGK in the presence of ATP, ADP or without effector molecules. Under any of these conditions, complex formation could be detected, but the affinity of the phosphomimetic variant to NAGK was about 10-20 fold lower than that of wild-type PII. By SPR, NAGK interaction with the PII-S49E was undetectable and could so-far only be observed by FRET analysis ${ }^{12}$. The respective dissociation constants are shown in Table 1. The PII-I86N-LgBiT has a single amino acid replacement, IIe86 to Asp86, which leads to constitutive high activation of NAGK. In agreement, the NanoBiT analysis revealed high affinity to NAGK, and importantly, the affinity remained high in the presence of ADP.

Inhibitory effect of arginine on PII-NAGK interaction. Another effector molecule that interferes with the PII-NAGK complex is arginine. Arginine binds to allosteric sites in NAGK, one site per each subunit, which are occupied in an anti-cooperative manner, thereby inhibiting its activity ${ }^{21,31}$. In complex with PII, NAGK feedback-inhibition by arginine is strongly relieved, presumably because the PII complex lowers the affinity of Arg to the allosteric binding sites. To find out how arginine, conversely, affects NAGK-SmBiT-PII-LgBiT complex formation, arginine was titrated to PII-LgBiT-NAGK-SmBiT complexes and luminescence was recorded. As shown in Fig. S1, very low concentrations of arginine slightly enhanced complex formation, but with increasing concentrations of Arg (when $2 \mathrm{mM}$ ATP or $1 \mathrm{mM}$ ATP/1 mM ADP were present), the luminescence signal decreased gradually to a new steady state level of approx. $60 \%$ of the initial value. The same experiment in presence of $2 \mathrm{mM}$ ADP revealed a slightly increasing luminescence signal at low Arg levels, which returned back to 
a

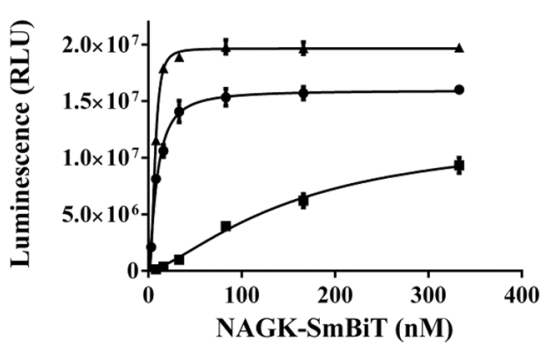

C

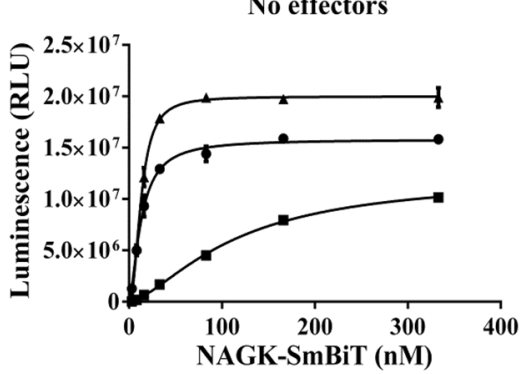

b
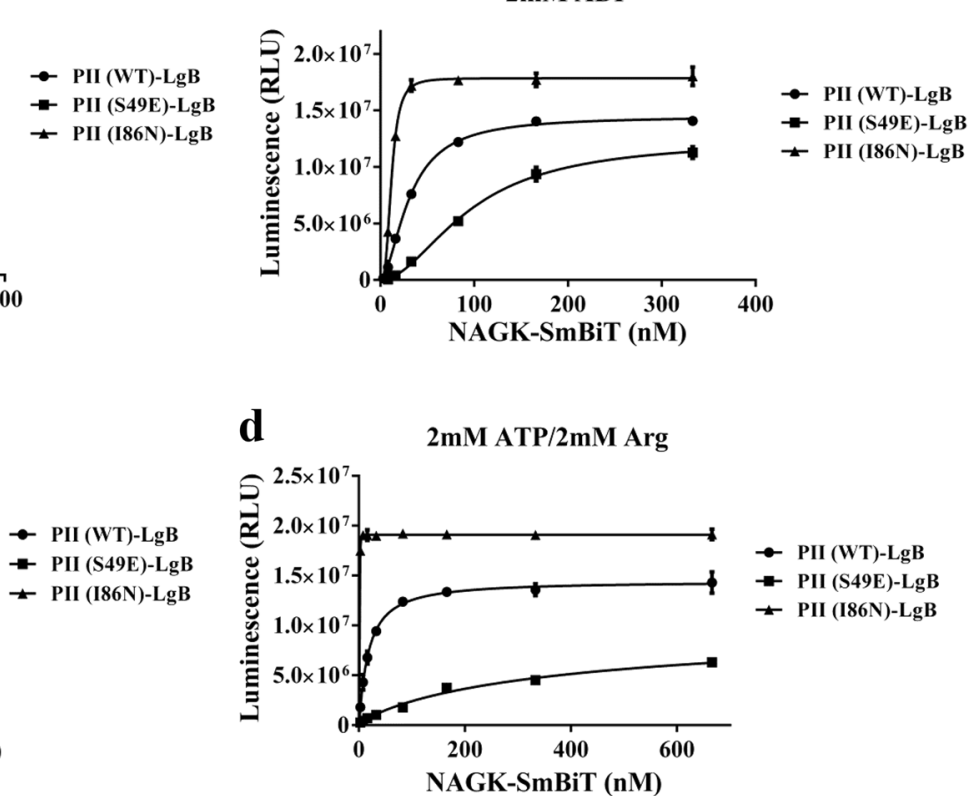

d



Figure 6. Determination of complex formation between PII-LgBiT variants (WT, S49E, I86N) and NAGKSmBiT measured by NanoBiT-sensor. (a) NAGK-SmBiT titration in the range of 3-333 nM with $10 \mathrm{pM}$ PII-LgBiT variants (WT, S49E, I86N) in the presence of $2 \mathrm{mM}$ ATP. (b) NAGK-SmBiT titration in the range of 3-333 nM with $10 \mathrm{pM}$ PII-LgBiT variants (WT, S49E, I86N) in the presence of $2 \mathrm{mM}$ ADP. (c) NAGK-SmBiT titration in the range of 3-333 nM with $10 \mathrm{pM}$ PII-LgBiT variants (WT, S49E, I86N) without effector molecules. (d) NAGK-SmBiT titration in the range of 3-666 nM with $10 \mathrm{pM}$ PII-LgBiT variants (WT, S49E, I86N) in the presence of $2 \mathrm{mM}$ ATP and $2 \mathrm{mM}$ Arginine. Graphs represent mean \pm SD of three independent experiments.

initial value at higher concentrations of Arg. In the absence of adenylnucleotides, low concentration of Arg were unable to increase complex formation and the luminescence signal (Fig. S1). These results indicate that the completely arginine-occupied NAGK has a reduced affinity for PII, whereas binding of Arg to the first allosteric site transiently increases the affinity. At a concentration of $2 \mathrm{mM}$ arginine, arginine completely inhibits the activity of free NAGK and inhibits the activity of NAGK in PII-complex to about $90 \%$. To determine the binding affinity of NAGK to PII under these assay conditions, increasing concentrations of NAGK were titrated to $10 \mathrm{pM}$ of the respective PII-LgBiT variants (Fig. 6d, Quantitative data are shown in Table 1. The affinity of PII was approx twofold lower in presence of $2 \mathrm{mM}$ Arg, in agreement with the reduced luminescence signal observed in the titration experiment with increasing Arg concentrations (Fig. S1). Obviously, the inhibition of enzyme activity is not caused by complex dissociation. Rather, it seems that PII can still bind to completely Arg-saturated NAGK, but the complex likely adopts a different conformation, which obstructs the catalytic activation of NAGK by PII.

Interestingly, Arg had a strong, differential effect on the affinity of NAGK towards different PII variants: Whereas on wild-type PII, it moderately reduced the affinity, an even stronger reduction was observed for the PII-S49E variant. Strikingly, the affinity towards PII-I86N variant was highly increased (Fig. 6d). This explains the strong stimulation of arginine synthesis in a Synechocystis strain, in which the PII wild-type protein was replaced by the PII-I86N variant, causing constitutive arginine overproduction and accumulation of cyanophycin ${ }^{32}$.

Feed-forward regulation of N-acetylglutamate (NAG) on PII-NAGK complex formation. Next, we investigated how the substrate of NAGK, N-acetylglutamate (NAG), affects its interaction with PII. The influence of the substrate on the interaction of NAGK with PII was so-far never investigated, but must be taken into consideration for understanding the in vivo situation. NAG was titrated into a binding assay, whereby NAGK was used at a concentration equivalent to half $\mathrm{Km}$, to enable the sensitive detection of changing affinity. As shown in Fig. 7a, increasing NAG concentrations caused a dramatic increase in PII-NAGK interaction. The binding constants were determined for a low $(2 \mathrm{mM})$ and a high $(50 \mathrm{mM})$ NAG concentration. $50 \mathrm{mM}$ NAG is the concentration of NAG, routinely used in enzyme assays. The results are displayed in Fig. 7b,C and Table 1. NAG stimulates PII-NAGK interaction under any conditions. In the presence of ATP the affinity doubles with $2 \mathrm{mM}$ NAG and increases more than tenfold with $50 \mathrm{mM}$ NAG (as compared to the absence of NAG). The stimulating effect of NAG on PII-NAGK interaction is so strong that efficiently stabilizes the complex in presence of ADP (see Fig. 7d) (20-fold increase with 50 mM NAG, Table 1). The presence of Arg mitigated the strong stimulating effect of NAG on PII-NAGK affinity. In presence of $2 \mathrm{mM}$ Arg, only a moderate increase by NAG on PII-NAGK interaction was detected (Fig. S2 and Table 1).

Interestingly, the S49E PII variant strongly bound to NAGK in the presence of 50 mM NAG and ATP, although this variant is unable to relieve NAGK from arginine inhibition (Fig. 7c). These results clearly demonstrate binding of PII to NAGK is not sufficient to cause a relief from Arg inhibition. The sophisticated hydrogen-bonding network organized by the $-\mathrm{OH}$ group of Ser49, which propagates into the catalytic centre of NAGK ${ }^{6}$ is required 
a

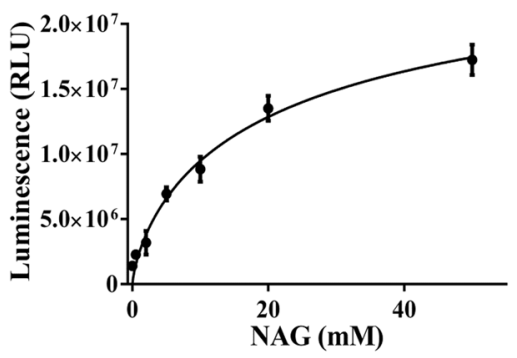

c

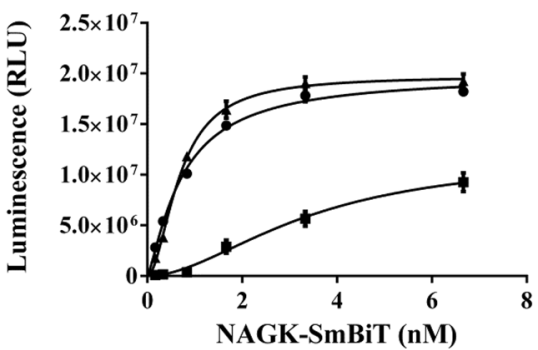

b
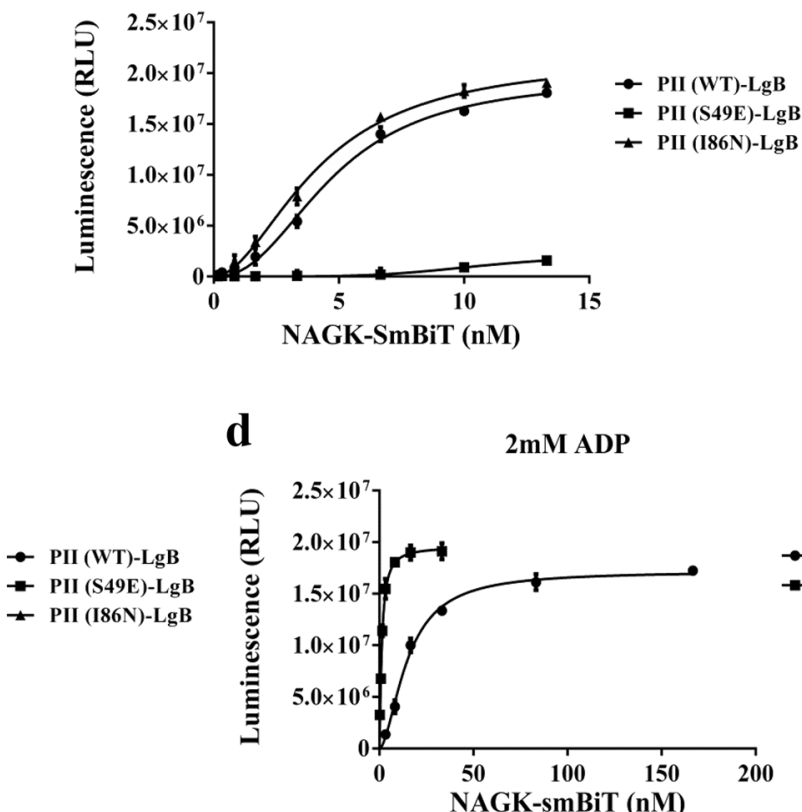

d

2mM ATP/2mM NAG

NAGK-SmBiT (nM)

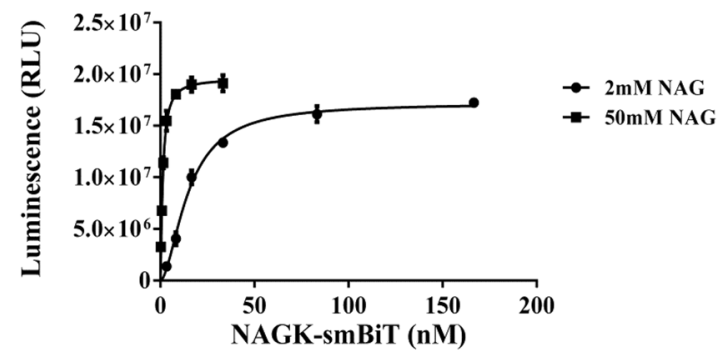

Figure 7. Effect of NAG on PII-NAGK interaction. (a): NAG titration (0-50 mM) for $10 \mathrm{pM}$ PII-LgBiT and $5 \mathrm{nM}$ NAGK-SmBiT in the presence of $2 \mathrm{mM}$ ATP. (b): NAGK-SmBiT titration to $10 \mathrm{pM}$ PII-LgBiT variants in the presence of $2 \mathrm{mM}$ ATP/ $2 \mathrm{mM}$ NAG. (c): NAGK-SmBiT titration to $10 \mathrm{pM}$ PII-LgBiT variants in the presence of $2 \mathrm{mM}$ ATP/ $50 \mathrm{mM}$ NAG. (d): NAGK-SmBiT titration to $10 \mathrm{pM}$ PII-LgBiT in the presence of $2 \mathrm{mM}$ ADP with either $2 \mathrm{mM}$ NAG or $50 \mathrm{mM}$ NAG. Graphs represent mean \pm SD of three independent experiments.

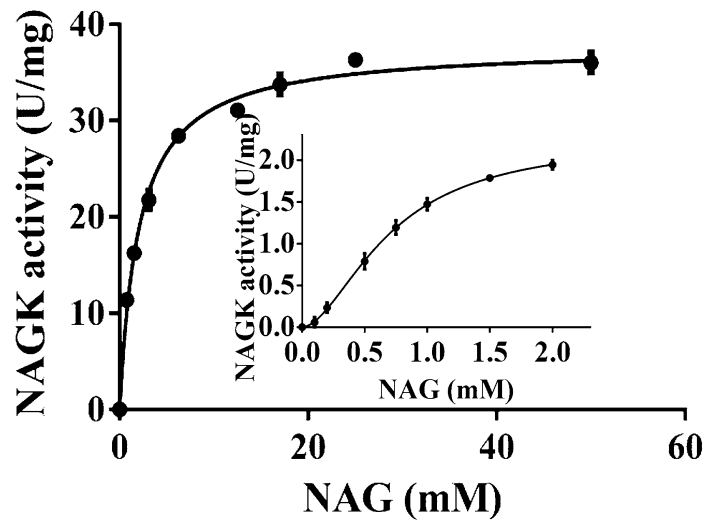

Figure 8. Activation of catalytic activity of NAGK by NAG in standarad NAGK assay and coupled NAGK assay. Standarad NAGK assay with NAG titration (0-50 mM) PII (1.2 $\mu \mathrm{g})$ in the form of complex with NAGK $(2 \mu \mathrm{g})$ in the presence of $10 \mathrm{mM}$ ATP. Coupled NAGK assay with NAG titration $(0-2 \mathrm{mM})$ for PII $(2.4 \mu \mathrm{g})$ in the form of complex with NAGK $(6 \mu \mathrm{g})$ in the presence of $1 \mathrm{mM}$ ATP/1 mM ADP and $0.1 \mathrm{mM}$ Arginine. Graphs represent mean \pm SD of three independent experiments.

to tune the enzyme. Therefore, the weak interaction of the S49E PII variant with NAGK does not affect enzyme activity. Under in vivo conditions, the concentration of NAG is probably in the low millimolar range (as deduced from the low abundance in metabolome data, e.g. ${ }^{17}$. Hence, the dependence of PII-NAGK complex formation on NAG should have physiological consequences.

With increasing substrate concentrations, the affinity of NAGK for PII binding increases, leading to enhanced kinetic activation. Therefore, we tested whether NAGK activity in the PII complex at low NAG concentration and with $1 \mathrm{mM}$ ATP/1 mM ADP and $0.1 \mathrm{mM}$ Arg follows a sigmoidal curve, and in comparison, performed the standard assay in presence of $10 \mathrm{mM}$ ATP (Fig. 8). The inset in Fig. 8 shows the result of the complex metabolite mixture. In fact, substrate-dependent activity follows a sigmoidal curve that could be fitted to a Hill slope of $1.76 \pm 0.032$. The increasing affinity of PII-NAGK with increasing concentrations of NAG results in a unique feed-forward like activation of the PII-NAGK complex by the enzyme substrate. 


\section{Conclusions}

The present analysis has established that NanoBiT is a reliable approach for the analysis of PII - receptor interactions. For the first time, we were able to accurately determine the affinity constants of PII with its target PipX and NAGK under identical experimental conditions.

The assay is robust and requires only minute amounts of protein. In contrast to surface plasmon resonance or Bio-layer interferometry analysis, the interaction occurs with proteins in solution. The only unnatural feature is the fusion of $\mathrm{LgBiT}$ and SmBiT fragments to the proteins of interest. Of note, the intrinsic affinity for LgBiT and $\mathrm{SmBiT}$ is extremely low through optimization of NanoBiT system, achieving a $\mathrm{K}_{\mathrm{D}}$ value as high as 190 $\mu \mathrm{M}^{25}$. Therefore the weak association between two parts of sensor should not influence the affinity of interacting proteins which is by several orders of magnitude higher (PII-PipX and PII-NAGK complexes in our study with $\mathrm{K}_{\mathrm{D}}$ values in the range of $\mathrm{nM}$ ). The specificity of association through PII interactions is also clearly indicated by the observation of fast dissociation of the PII-PipX complex upon addition of 2-OG. (See Fig. 3c): If the LgBiTSmBiT fragments would affect the affinity, no such rapid dissociation would be visible. The intrinsic affinity is therefore so low that it doesn't affect the experimentally measured affinities between PII and its partner proteins. In accord, we observed almost negligible background under our experimental conditions, probably due to the low concentration of proteins used, far below the intrinsic affinities to Lg-BiT and Sm-BiT. Therefore, the emergence of luminescence can safely be interpreted as indication of complex formation.

In previous experiments on cyanobacterial PII protein interactions by Surface-Plasmon-Resonance (SPR), the $\mathrm{K}_{\mathrm{D}}$ values for the PII-NAGK and PII-PipX interactions were never reported (the assays were carried out with the aim to determine the relative influence of effector molecules on PII interactions). The only $\mathrm{K}_{\mathrm{D}}$ value for the PII-NAGK complex was determined previously by ultrafiltration experiments in presence of very high NAGK concentrations, so that complex formation equilibrium was almost completely on the side of the complex. From the small amount of free PII a $K_{D}$ of approximately $73 \mathrm{nM}$ was reported ${ }^{6}$. The $K_{D}$ value that we here obtained by NanoBiT system $\left(K_{D}=12.9 \mathrm{nM}\right)$ is almost six times lower although still in the same magnitude of order. In principle, the difference could be caused by different buffers used in these experiments. Very recently, we applied Biolayer interferometry, to measure PII interactions, whereby the interaction of PII with NAGK was tested as a proof of principle for the method (Supplementary material in Scholl et al.) ${ }^{17}$. Therefore, we now re-calculated the binding curves using curve fitting from association and dissociation kinetics according to Octet data analysis $\mathrm{HT}$ software (ForteBio) and this results in an apparent $\mathrm{K}_{\mathrm{D}}$ of $11.7 \pm 0.005 \mathrm{nM}$ which is perfect agreement with the NanoBiT sensor data with the $K_{D}=12.9 \pm 0.65 \mathrm{nM}$. This gives additional credibility to the results from the NanoBit sensor.

Due to the high sensitivity of the interaction assay, it was even possible to detect residual low-affinity interactions, such as those between the PII variant PII-S49E with NAGK, which had never been detected before with SPR or BLI analysis. From the crystal structure of the PII-NAGK complex it is known that the seryl-residue at the tip of the T-loop stabilizes the tight PII-NAGK complex and causes the enzymatic activation of NAGK by PII. A weak interaction of the PII S49 variant (PII-S49G) could, however, be detected by FRET analysis ${ }^{12,13}$ and was interpreted as indication of the transient encounter complex that is formed between PII and NAGK. This encounter complex was already predicted from the analysis of PII and NAGK mutants ${ }^{20}$. The NanoBiT study now for the first time resolved the affinity of the encounter complex, and revealed that this complex is not favoured by the state of adenyl-nucleotide binding to PII, as can be deduced from the dissociation constants of PII-S49ENAGK in presence of different effectors. The PII-S49E variant represents a phosphomimetic variant of PII: wildtype PII is phosphorylated at S49 under nitrogen-poor conditions. The phopshomimetic variant is not able to catalytically activate NAGK, and it is reasonable to assume that likewise, also phospho-PII will not activate PII. However, the result of the NanoBiT analysis shows that the complex doesn't dissociate completely, suggesting that upon phosphorylation of PII at the tip of the T-loop, the proteins remain loosely associated. Dephosphorylation of PII under appropriate condition would then enable an immediate re-formation of the PII-NAGK complex.

A further application of the PII-based NanoBiT toolbox is the quantification of competitive binding of different PII receptors to PII. Here, we have demonstrated adenyl-nucleotide-dependent competition between PipX and NAGK for PII binding. PII has the highest affinity for PipX in the presence of ADP (5.7 nM), whereas under these conditions, affinity for NAGK is low $(30.8 \mathrm{nM})$. Therefore, not surprisingly, NAGK was not able to compete with PipX: binding of PipX-SmBiT was unaffected by the addition of NAGK. By contrast, in presence of ATP, affinity of PII to PipX is diminished $(52.4 \mathrm{nM})$ concomitantly with an increased affinity for NAGK $(8.8 \mathrm{nM})$. Under these conditions, addition of NAGK lowered PII-LgBiT-PipX-SmBiT interaction, reflecting successful competition. Surprisingly, however, even a large excess of NAGK was unable to abolish the signal of the PII-LgBi-PipX-SmBiT sensor pair, although PII should have been almost completely saturated with NAGK in these conditions. The remaining luminescence signal could indicate vicinity between PipX-SmBiT and PIILgBiT bound to NAGK, but the type of interaction remains to be clarified.

Altogether, this study has demonstrated the usefulness of the NanoBiT technology to study the interactions between PII and its receptors, and in a general sense, to investigate weak and transient protein-protein interactions. Future studies will transfer the method to the analysis of the recently identified PII receptors to gain quantitative insights in PII interactions with the ultimate goal to be able to model the network of PII interactions, for which quantitative data are required.

\section{Methods}

Cloning and DNA purification. To generate the split NanoLuc sensor, PII-LgBiT, PipX-SmBiT and NAGKSmBiT synthetic genes, which already contained overlapping sequences for Gibson assembly, were ordered from Integrated DNA Technologies (Biotechnology company, Leuven, Belgium) (Table S1). PII- LgBiT was cloned in pASK-IBA3 vector linearized by EcoR1 and HindIII. Two other constructs, PipX-SmBiT and NAGK-SmBiT 
were cloned into NdeI and BamHI sites of pTEV5 vector with $\mathrm{N}$-terminal-fused $\mathrm{His}_{6}$-tag via Gibson cloning as described previously ${ }^{33}$. Plasmid DNA was amplified by transformation into $E$. coli DH1O $\beta$, which was grown at $37^{\circ} \mathrm{C}$ overnight on agar media containing the appropriate antibiotic for selection. Positive colonies were identified by colony PCR and then transferred to Lysogeny broth (LB) supplemented with the appropriate antibiotic and grown at $37^{\circ} \mathrm{C}$ overnight. Plasmid DNA was purified from cells using the Monarch Plasmid Miniprep Kit (New England Biolabs, Frankfurt a.M., Germany) according to the manufacturer's instructions. All sequences were verified using the GATC LIGHTRUN service (Eurofins Genomics, Ebersberg, Germany). Afterwards, the resulting plasmids were transformed into electrocompetent E. coli Lemo21 cells for subsequent expression of recombinant proteins.

Overexpression and Purification of recombinant proteins. The expression of recombinant proteins was performed by growing the cells at $37^{\circ} \mathrm{C}$ under continuous shaking at $120 \mathrm{rpm}$ to an OD600 of about 0.6-0.8. Depending on the plasmid the appropriated amount of the antibiotic was added to the culture. Overexpression was induced by the addition of $0.5 \mathrm{mM}$ isopropyl- $\beta$-D-thiogalactopyranoside for His-tagged proteins and $0.2 \mu \mathrm{g} / \mathrm{ml}$ of anhydrotetracycline for Strep-tagged proteins. Induction was performed at $20^{\circ} \mathrm{C} / 120 \mathrm{rpm}$ overnight. Then, the cells were harvested on the next morning by centrifugation $\left(3.500 \mathrm{rpm}\right.$ for $15 \mathrm{~min}$ at $\left.4^{\circ} \mathrm{C}\right)$ and pellets were stored at $-20^{\circ} \mathrm{C}$ until use.

The recombinant protein (PII) containing the strep ${ }_{10}$-tag was purified via $5 \mathrm{ml}$ Strep-tactin ${ }^{\star}$ superflow columns. The cells were lysed in $50 \mathrm{ml}$ lysis buffer containing $50 \mathrm{mM}$ Tris/ $\mathrm{HCl}$ (pH 7.4), $50 \mathrm{mM} \mathrm{KCl,} 5 \mathrm{mM} \mathrm{MgCl}_{2}$, $2 \mathrm{mM}$ EDTA, $2 \mathrm{mM}$ DTT and $0.5 \mathrm{mM}$ PMSF. Ultrasonication was applied to disrupt the cells and lysate was centrifuged for $45 \mathrm{~min}$ at $55,000 \times \mathrm{g}$ at $4^{\circ} \mathrm{C}$. The supernatant was loaded on a $5 \mathrm{ml}$ of Strep-Tactin Superflow column (IBA, Göttingen, Germany) that was equilibrated with the appropriate lysis buffer before use. Next, the column was washed with $100 \mathrm{ml}$ of Strep washing buffer containing $100 \mathrm{mM}$ Tris/ $\mathrm{HCl}(\mathrm{pH} 8.0), 150 \mathrm{mM} \mathrm{NaCl}$, $1 \mathrm{mM}$ EDTA and then it was eluted with Strep elution buffer containing $100 \mathrm{mM}$ Tris/ $\mathrm{HCl}(\mathrm{pH} 8.0), 150 \mathrm{mM}$ $\mathrm{NaCl}, 1 \mathrm{mM}$ EDTA, $2.5 \mathrm{mM}$ D-desthiobiotine. Roti-Quant (Roth, Karlsruhe, Germany) was applied to estimate the concentration of the eluted proteins in the elution fractions by following the manufacturer's instructions. The eluted protein was loaded on SDS-PAGE to check the purity and size of protein. The recombinant PII protein

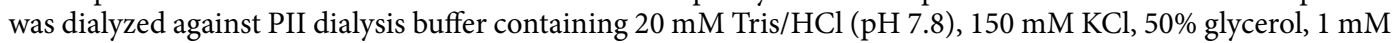
DTT and stored at $-20^{\circ} \mathrm{C}$ until use.

The recombinant proteins (PipX and NAGK) containing His $_{6}$-tags were purified via $1 \mathrm{ml} \mathrm{Ni-NTA} \mathrm{HisTrap}$ columns. The purification steps were similar to those of strep tag-proteins, while the buffers and columns were different. The lysis buffer contained $50 \mathrm{mM}$ Tris/ $\mathrm{HCl}(\mathrm{pH} 7.4$ ), $50 \mathrm{mM} \mathrm{KCl,} 5 \mathrm{mM} \mathrm{MgCl}, 10 \mathrm{mM}$ Imidazole, $2 \mathrm{mM}$ DTT and $0.2 \mathrm{mM}$ PMSF. HisTrap columns were washed with $100 \mathrm{ml}$ of His washing buffer 1 including $50 \mathrm{mM} \mathrm{NaH}_{2} \mathrm{PO}_{4}$ (pH 8.0), $300 \mathrm{mM} \mathrm{NaCl}, 20 \mathrm{mM}$ Imidazole, followed by $50 \mathrm{ml}$ of His washing buffer 2 with $50 \mathrm{mM} \mathrm{NaH}_{2} \mathrm{PO}_{4}$ (pH 8.0), $300 \mathrm{mM} \mathrm{NaCl}, 40 \mathrm{mM}$ Imidazole. Elution of the bound proteins from the column with Ni-NTA was performed with an elution buffer containing $50 \mathrm{mM} \mathrm{NaH}_{2} \mathrm{PO}_{4}$ (pH 8.0), $300 \mathrm{mM} \mathrm{NaCl}$, $250 \mathrm{mM}$ Imidazole. The fractions consisting of recombinant protein were dialyzed in the respective dialysis buffer containing $50 \mathrm{mM}$ Tris/ $\mathrm{HCl}$ (pH 7.8), $100 \mathrm{mM} \mathrm{KCl,} 5 \mathrm{mM} \mathrm{MgCl}$, $0.5 \mathrm{mM}$ EDTA, $1 \mathrm{mM}$ DTT and 50\% glycerol. The proteins were stored at $-20^{\circ} \mathrm{C}$ for further use.

Establishment of the bioluminescence assay. The luminescence assay for both types of measurementtime point and time course-was performed in a buffer containing $50 \mathrm{mM}$ Tris/ $\mathrm{HCl}$ (pH 7.6), $100 \mathrm{mM} \mathrm{KCl,} \mathrm{10 \%}$ glycerol, $2 \mathrm{mM} \mathrm{MgCl}_{2}$ and $0.1 \%$ BSA. Next, the sensor proteins were added to the final concentration of $10 \mathrm{pM}$ (trimer) for PII-LgBiT and a range of concentrations (0-800 nM) for PipX-SmBiT. The respective concentrations of effector metabolites (ATP, ADP, and ATP/2-OG) were included to the final volume (500 $\mu \mathrm{l})$ in the test tubes. The mixture was incubated for $15 \mathrm{~min}$ at $30^{\circ} \mathrm{C}$. Afterwards, $0.5 \mu$ of Nano-Glo Luciferase substrate (Promega, Walldorf, Germany) per $500 \mu \mathrm{l}$ of total volume was added to each reaction tube. The solution was incubated for $5 \mathrm{~min}$ at room temperature to allow the reagent and proteins to adapt to the buffer. Subsequently the luminescence was quantified in a luminometer (Sirus Luminometer, Berthold Detection System, Germany) for $10 \mathrm{~s}$ with $10 \mathrm{~s}$ delay. The recorded luminescence is reported as relative light units (RLU). For background value, every protein was measured separately. Then, the recorded signal was subtracted from the luminescence of the forming complex.

To investigate the subsequent effect of metabolites in time-course experiments for PII-LgBiT and PipXSmBiT, 10 pM PII was added to $10 \mathrm{nM}$ PipX-SmBiT in the above mentioned buffer. Then, $0.5 \mu$ l of Nano-Glo ${ }^{\circ}$ Luciferase substrate was added to the reaction tube. After $5 \mathrm{~min}$ incubation, the luminescence was measured via FB12 Sirius software with 20 s intervals in the Sirus luminometer for some minutes. To monitor the effect of metabolites on complex formation, the appropriate amounts were added to the reaction tube and the measurement was continued for several minutes until a maximum binding was reached.

In the case of luminescence measurement (time point) for PII-LgBiT variants and NAGK-SmBiT, the respective concentration of proteins and metabolites were incubated for $40 \mathrm{~min}$ at $30^{\circ} \mathrm{C}$. The rest of the procedure was the same as the one described above.

Competition assay based on NanoBiT sensor. Competition assay between PipX and NAGK-SmBiT to bind to PII-LgBiT in the presence of $2 \mathrm{mM}$ effector molecules, was performed with incubation of $10 \mathrm{pM}$ PIILgBiT trimer and different concentration of PipX (180, 900 and $1800 \mathrm{nM})$ monomer for $15 \mathrm{~min}$ at $30^{\circ} \mathrm{C}$. Then a constant concentration of NAGK-SmBiT $(180 \mathrm{nM})$ monomer was added to the mixure and incubation was continued for other $30 \mathrm{~min}$ at $30^{\circ} \mathrm{C}$. Later, $0.5 \mu \mathrm{l}$ of Nano-Glo Luciferase substrate (Promega, Walldorf, Ger- 
many) per $500 \mu \mathrm{l}$ of total volume was added to each reaction tube. After $5 \mathrm{~min}$ of incubation, luminescence was quantified in a luminometer (Sirus Luminometer, Berthold Detection System, Germany) for $10 \mathrm{~s}$ with $10 \mathrm{~s}$ delay.

Competition assay between NAGK and PipX-SmBiT to bind to PII-LgBiT was performed with incubation of 10 pM PII-LgBiT trimer and different concentration of NAGK (180, 900, 1800 and $3600 \mathrm{nM})$ monomer for $30 \mathrm{~min}$ at $30^{\circ} \mathrm{C}$. Then a constant concentration of PipX-SmBiT $(10 \mathrm{nM})$ monomer was added to the mixture and incubation was continued for other $15 \mathrm{~min}$ at $30^{\circ} \mathrm{C}$. The final steps were performed as described above.

AGPR coupled NAGK activity assay. To study the effect of NAG on the activity of the $\mathrm{P}_{\mathrm{II}}$-NAGK complex, the specific activity of NAGK from Synechocystis sp. PCC 6803 was assayed by coupling NAGK-dependent NAG phosphorylation to an auxiliary enzyme (AGPR) from E. coli. AGPR reduced the NAG-phosphate by using NADPH as reductant and the change was recorded at $340 \mathrm{~nm}$ as described ${ }^{34,35}$. The reaction buffer consisted of $50 \mathrm{mM}$ imidazole (pH 7.5), $50 \mathrm{mM} \mathrm{KCl,} 20 \mathrm{mM} \mathrm{MgCl}_{2}, 0.2 \mathrm{mM}$ NADPH, $0.5 \mathrm{mM}$ DTT, $1 \mathrm{mM}$ ATP, $1 \mathrm{mM}$ ADP and $0.1 \mathrm{mM}$ arginine. Each reaction contained $10 \mu \mathrm{g}$ AGPR, $2.4 \mu \mathrm{g}$ PII and $6 \mu \mathrm{g}$ NAGK with different concentration of NAG $(0-2 \mathrm{mM})$. The reaction was started by the addition of NAGK. Thereby, oxidation of one molecule of NADPH was recorded over a period of $10 \mathrm{~min}$ with a spectrophotometer (SPECORD 200, Analytik Jena). The reaction velocity was calculated with molar absorption of NADPH of $\Sigma_{340}=6178 \mathrm{~L} \mathrm{~mol}^{-1} \mathrm{~cm}^{-1}$ from the slope of the change of absorbance per time.

Standard NAGK activity assay. To assess the activity of NAGK, the ADP production was coupled to the $\mathrm{NADH}$ oxidation using the auxiliary enzymes pyruvate kinase and lactate dehydrogenase according to previously described ${ }^{35,36}$. The activity of NAGK was expressed in $\mu \mathrm{mol} / \mathrm{min} / \mathrm{mg}$.

Statistical analysis. The results were performed three times to confirm reproducibility. They were expressed as the mean \pm SEM of the indicated experiment. The FB12 Sirius software was used to record the luminescence in time-course experiments. Then, statistical analysis was performed by applying GraphPad Prism 6 with fitting curve "one site-specific bind with Hill slope".

Received: 23 March 2021; Accepted: 24 May 2021

Published online: 15 June 2021

\section{References}

1. Selim, K. A., Ermilova, E. \& Forchhammer, K. From cyanobacteria to Archaeplastida: new evolutionary insights into PII signalling in the plant kingdom. New Phytol. 227, 722-731 (2020).

2. Forchhammer, K. \& Selim, K. A. Carbon/nitrogen homeostasis control in cyanobacteria. FEMS Microbiol. Rev. 44, 33-53 (2020).

3. Gerhardt, E. C. et al. The protein-protein interaction network reveals a novel role of the signal transduction protein PII in the control of c-di-GMP homeostasis in Azospirillum brasilense. Msystems 5, 10 (2020).

4. Burillo, S., Luque, I., Fuentes, I. \& Contreras, A. Interactions between the nitrogen signal transduction protein PII and N-acetyl glutamate kinase in organisms that perform oxygenic photosynthesis. J. Bacteriol. 186, 3346-3354 (2004).

5. Heinrich, B. Bumblebee Economics (Harvard University Press, 2004).

6. Llácer, J. L. et al. The crystal structure of the complex of PII and acetylglutamate kinase reveals how PII controls the storage of nitrogen as arginine. Proc. Natl. Acad. Sci. 104, 17644-17649 (2007).

7. Llácer, J. L. et al. Structural basis for the regulation of NtcA-dependent transcription by proteins PipX and PII. Proc. Natl. Acad. Sci. 107, 15397-15402 (2010).

8. Espinosa, J., Forchhammer, K., Burillo, S. \& Contreras, A. Interaction network in cyanobacterial nitrogen regulation: PipX, a protein that interacts in a 2-oxoglutarate dependent manner with PII and NtcA. Mol. Microbiol. 61, 457-469 (2006).

9. Fokina, O., Chellamuthu, V.-R., Zeth, K. \& Forchhammer, K. A novel signal transduction protein PII variant from Synechococcus elongatus PCC 7942 indicates a two-step process for NAGK-PII complex formation. J. Mol. Biol. 399, 410-421 (2010).

10. Fokina, O., Herrmann, C. \& Forchhammer, K. Signal-transduction protein PII from Synechococcus elongatus PCC 7942 senses low adenylate energy charge in vitro. Biochem. J. 440, 147-156 (2011).

11. Zeth, K., Fokina, O. \& Forchhammer, K. Structural basis and target-specific modulation of ADP sensing by the Synechococcus elongatus PII signaling protein. J. Biol. Chem. 289, 8960-8972 (2014).

12. Lüddecke, J. \& Forchhammer, K. From P II signaling to metabolite sensing: a novel 2-oxoglutarate sensor that details P II-NAGK complex formation. PLoS One 8, e83181 (2013).

13. Lüddecke, J. \& Forchhammer, K. Energy sensing versus 2-oxoglutarate dependent ATPase switch in the control of Synechococcus P II interaction with its targets NAGK and PipX. PLoS One 10, e0137114 (2015).

14. Chen, H. L., Bernard, C. S., Hubert, P., My, L. \& Zhang, C. C. Fluorescence resonance energy transfer based on interaction of PII and PipX proteins provides a robust and specific biosensor for 2-oxoglutarate, a central metabolite and a signalling molecule. FEBS J. 281, 1241-1255 (2014).

15. Hauf, W., Watzer, B., Roos, N., Klotz, A. \& Forchhammer, K. Photoautotrophic polyhydroxybutyrate granule formation is regulated by cyanobacterial phasin PhaP in Synechocystis sp. strain PCC 6803. Appl. Environ. Microbiol. 81, 4411-4422 (2015).

16. Watzer, B. \& Forchhammer, K. Cyanophycin synthesis optimizes nitrogen utilization in the unicellular cyanobacterium Synechocystis sp. strain PCC 6803. Appl. Environ. Microbiol. 84, 25 (2018).

17. Scholl, J., Dengler, L., Bader, L. \& Forchhammer, K. Phosphoenolpyruvate carboxylase from the cyanobacterium Synechocystis sp. PCC 6803 is under global metabolic control by PII signaling. Mol. Microbiol. 114, 292-307 (2020).

18. Orthwein, T. et al. The novel PII-interacting regulator PirC identifies phosphoglycerate mutase as a key control point of carbon storage metabolism in cyanobacteria. Proc. Natl. Acad. Sci. 118 (6) e2019988118 (2021).

19. Bolay, P. et al. The novel PII-interacting protein PirA controls flux into the cyanobacterial ornithine-ammonia cycle. MBio 12 (2): e00229-21 (2021).

20. Fokina, O., Chellamuthu, V.-R., Forchhammer, K. \& Zeth, K. Mechanism of 2-oxoglutarate signaling by the Synechococcus elongatus PII signal transduction protein. Proc. Natl. Acad. Sci. 107, 19760-19765 (2010).

21. Rubio, V., Marco-Marín, C. \& Llácer, J. L. Nitrogen storage regulation by PII protein: lessons learned from taxonomic outliers. FEBS J. 287, 439-442 (2020). 
22. Lüddecke, J. et al. P II protein-derived FRET Sensors For Quantification And Live-Cell Imaging Of 2-Oxoglutarate. Sci. Rep. 7, $1-13$ (2017).

23. Remy, I. \& Michnick, S. W. A highly sensitive protein-protein interaction assay based on Gaussia luciferase. Nat. Methods 3, 977-979 (2006).

24. Hall, M. P. et al. Engineered luciferase reporter from a deep sea shrimp utilizing a novel imidazopyrazinone substrate. ACS Chem. Biol. 7, 1848-1857 (2012).

25. Dixon, A. S. et al. NanoLuc complementation reporter optimized for accurate measurement of protein interactions in cells. ACS Chem. Biol. 11, 400-408 (2016).

26. Promega. NanoBiT Protein:Protein Interaction System Technical Manual, https://www.promega.de/resources/protocols/technicalmanuals/101/nanobit-protein-protein-interaction-system-protocol/.

27. Tsang, T. F. et al. Simple method for studying in vitro protein-protein interactions based on protein complementation and its application in drug screening targeting bacterial transcription. ACS Infect. Dis. 5, 521-527 (2019).

28. Doello, S., Burkhardt, M. \& Forchhammer, K. The essential role of sodium bioenergetics and ATP homeostasis in the developmental transitions of a cyanobacterium. Curr. Biol. 31, 1606-1615 (2021).

29. Espinosa, J., Labella, J. I., Cantos, R. \& Contreras, A. Energy drives the dynamic localization of cyanobacterial nitrogen regulators during diurnal cycles. Environ. Microbiol. 20, 1240-1252 (2018).

30. Díaz-Troya, S., Roldán, M., Mallén-Ponce, M. J., Ortega-Martínez, P. \& Florencio, F. J. Lethality caused by ADP-glucose accumulation is suppressed by salt-induced carbon flux redirection in cyanobacteria. J. Exp. Bot. 71, 2005-2017 (2020).

31. Beez, S., Fokina, O., Herrmann, C. \& Forchhammer, K. N-acetyl-L-glutamate kinase (NAGK) from oxygenic phototrophs: PII signal transduction across domains of life reveals novel insights in NAGK control. J. Mol. Biol. 389, 748-758 (2009).

32. Watzer, B. et al. The signal transduction protein PII controls ammonium, nitrate and urea uptake in cyanobacteria. Front. Microbiol. 10, 1428 (2019).

33. Gibson, D. G. et al. Enzymatic assembly of DNA molecules up to several hundred kilobases. Nat. Methods 6, 343-345 (2009).

34. Takahara, K., Akashi, K. \& Yokota, A. Continuous spectrophotometric assays for three regulatory enzymes of the arginine biosynthetic pathway. Anal. Biochem. 368, 138-147 (2007).

35. Lapina, T., Selim, K. A., Forchhammer, K. \& Ermilova, E. The PII signaling protein from red algae represents an evolutionary link between cyanobacterial and Chloroplastida PII proteins. Sci. Rep. 8, 1-14 (2018).

36. Selim, K. A., Lapina, T., Forchhammer, K. \& Ermilova, E. Interaction of N-acetyl-l-glutamate kinase with the PII signal transducer in the non-photosynthetic alga Polytomella parva: Co-evolution towards a hetero-oligomeric enzyme. FEBS J. 287, 465-482 (2020).

\section{Acknowledgements}

We appreciate Dr. Libera Lo Presti for her helpful comments and proof-reading this manuscript.

\section{Author contributions}

R.R. developed the NanoBiT sensor and performed in vitro assays in bacteria. K.F. designed and supervised the experiments. R.R. and K.F. Analyzed the data and prepared the manuscript.

\section{Funding}

This project was supported by DFG Grant Fo195/21-1 and infrastructural support from DFG-funded EXC 2124 "Controlling Microbes to Fight Infections. Further, we acknowledge support by Open Access Publishing Fund of University of Tübingen. Open Access funding enabled and organized by Projekt DEAL.

\section{Competing interests}

The authors declare no competing interests.

\section{Additional information}

Supplementary Information The online version contains supplementary material available at https://doi.org/ 10.1038/s41598-021-91856-2.

Correspondence and requests for materials should be addressed to K.F.

Reprints and permissions information is available at www.nature.com/reprints.

Publisher's note Springer Nature remains neutral with regard to jurisdictional claims in published maps and institutional affiliations.

Open Access This article is licensed under a Creative Commons Attribution 4.0 International

License, which permits use, sharing, adaptation, distribution and reproduction in any medium or format, as long as you give appropriate credit to the original author(s) and the source, provide a link to the Creative Commons licence, and indicate if changes were made. The images or other third party material in this article are included in the article's Creative Commons licence, unless indicated otherwise in a credit line to the material. If material is not included in the article's Creative Commons licence and your intended use is not permitted by statutory regulation or exceeds the permitted use, you will need to obtain permission directly from the copyright holder. To view a copy of this licence, visit http://creativecommons.org/licenses/by/4.0/.

(c) The Author(s) 2021 